\title{
Correction to: Femtosecond X-ray induced electron kinetics in dielectrics: application for FEL-pulse-duration monitor
}

\author{
Nikita Medvedev ${ }^{1,2}$ (D)
}

Received: 31 January 2019 / Accepted: 29 March 2019 / Published online: 16 April 2019

(c) Springer-Verlag GmbH Germany, part of Springer Nature 2019

\section{Correction to: Appl. Phys. B (2015) 118:417-429 https://doi.org/10.1007/s00340-015-6005-4}

In the original paper, there was a mistype in Eq. (1), which instead should read:

$$
\begin{aligned}
\frac{\mathrm{d} \sigma}{\mathrm{d} w} & =S \sum_{n=1}^{3} F_{n}\left(\frac{1}{(1+w)^{n}}+\frac{1}{(K-w)^{n}}\right), \\
F_{1} & =-\frac{F_{2}}{K+1}, \quad F_{2}=\frac{1}{K+u+1}, \quad F_{3}=\frac{\ln (K)}{K+u+1}, \\
S & =4 \pi a_{0}^{2} N\left(\frac{R y}{I_{\mathrm{p}}}\right)^{2},
\end{aligned}
$$

where all the notations are the same as in the original work; note the change in $F_{1}$ term.

Equation (2) should be corrected as follows:

$$
\sigma=\frac{S}{K+u+1}\left[\frac{\ln (K)}{2}\left(1-K^{-2}\right)+\left(1-K^{-1}\right)-\frac{\ln (K)}{K+1}\right] .
$$

Note the minus sign inside of the first bracket instead of a mistyped plus in the original article.

The original article can be found online at https://doi.org/10.1007/ s00340-015-6005-4.

Nikita Medvedev

nikita.medvedev@fzu.cz

1 Institute of Plasma Physics CAS, v.v.i., Za Slovankou 3, 18200 Prague, Czech Republic

2 Institute of Physics CAS, v.v.i., Na Slovance 2, 18221 Prague, Czech Republic
Additionally, there was an unfortunate hard-to-notice minor bug in the impact ionization subroutine of the code that resulted in cascade durations being erroneously stretched in time by a factor of $\sim 1.5-2$. It does not affect the qualitative results reported, neither does it invalidate the conclusions-all the drawn conclusions remain unaffected (the proposed scheme for selection of optimal materials tailored for a particular FEL photon energy has recently been confirmed experimentally [1]). However, quantitatively, electronic cascades are shortened after the fix. The corrected figures can be found below.

I am grateful to S. Ashok (TU Kaiserslautern) for pointing out the misprints in the paper and for the help in identifying the bug in the code.

The densities of holes shown in Fig. 2 are a result of interplay of two processes: impact ionization that increases their number, and Auger decay that decreases it. Only the former one was affect by the bug in the code, which results in it now being faster with respect to the erroneous one in the original work; whereas the latter one has the same (correct) characteristic time as before. Thus, the overall shape of the curves is now slightly different. The same effect is seen in derivatives of the electron densities, Fig. 6, where the curves shapes are slightly altered. 


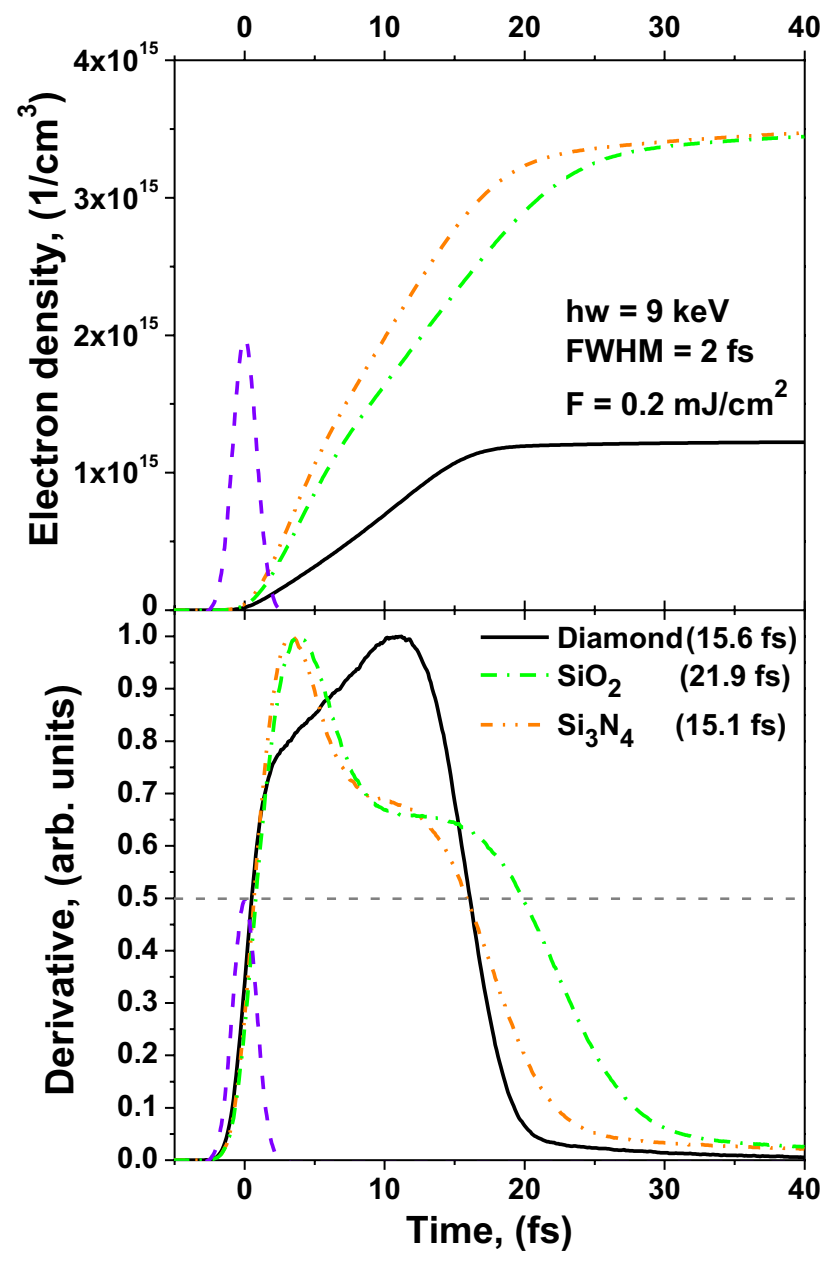

Update to Fig. 1 from the original article. Modeled electron density in diamond, $\mathrm{SiO}_{2}$ and $\mathrm{Si}_{3} \mathrm{~N}_{4}$ irradiated with a 2 fs laser pulse of $0.2 \mathrm{~mJ} /$ $\mathrm{cm}^{2}$ fluence and $9 \mathrm{keV}$ photon energy (top-panel), and the time derivative of the densities (bottompanel); extracted FWHM of the electron density increase is shown in the legend of the figure. Laser pulse envelope is schematically shown as a violet dashed line

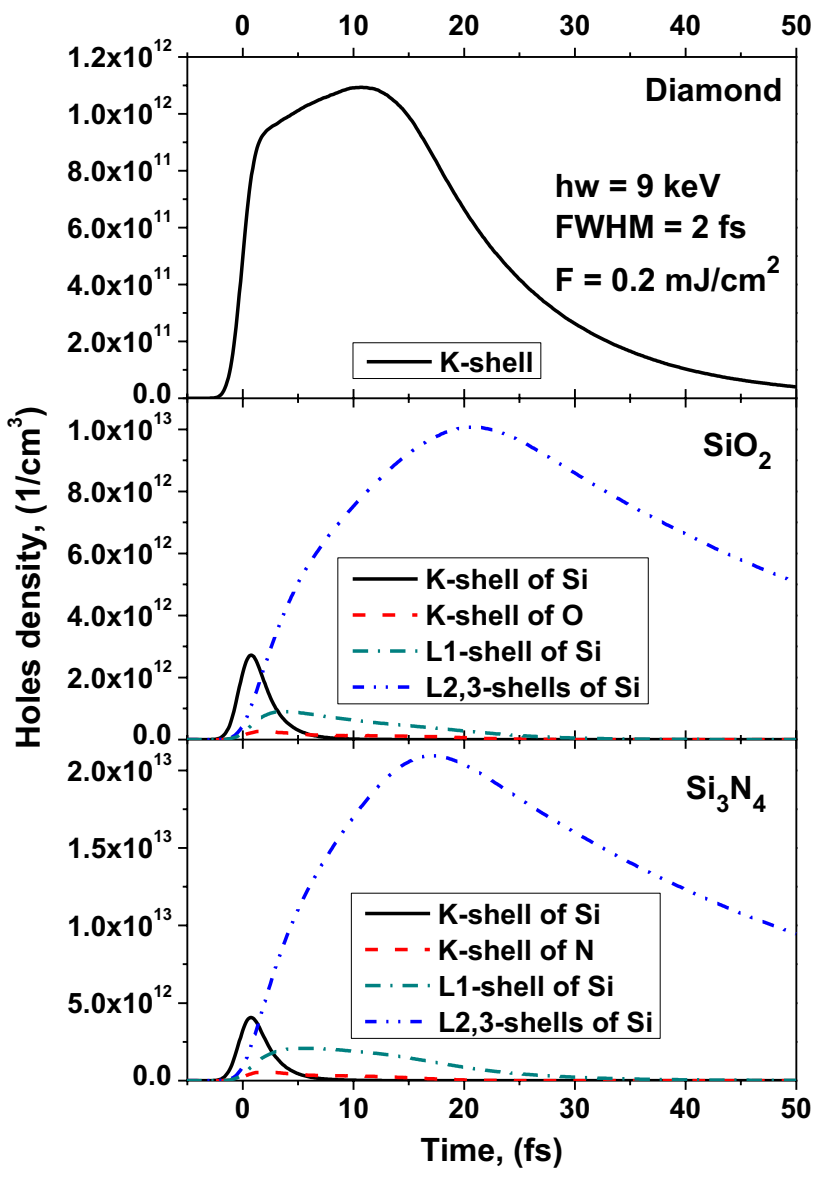

Update to Fig. 2 from the original article. Modeled density of core holes in different shells in diamond (top-panel), $\mathrm{SiO}_{2}$ (middle-panel) and $\mathrm{Si}_{3} \mathrm{~N}_{4}$ (bottom-panel) irradiated with a 2 fs laser pulse of $0.2 \mathrm{~mJ} /$ $\mathrm{cm}^{2}$ fluence and $9 \mathrm{keV}$ photon energy. Valence shells holes are not shown 


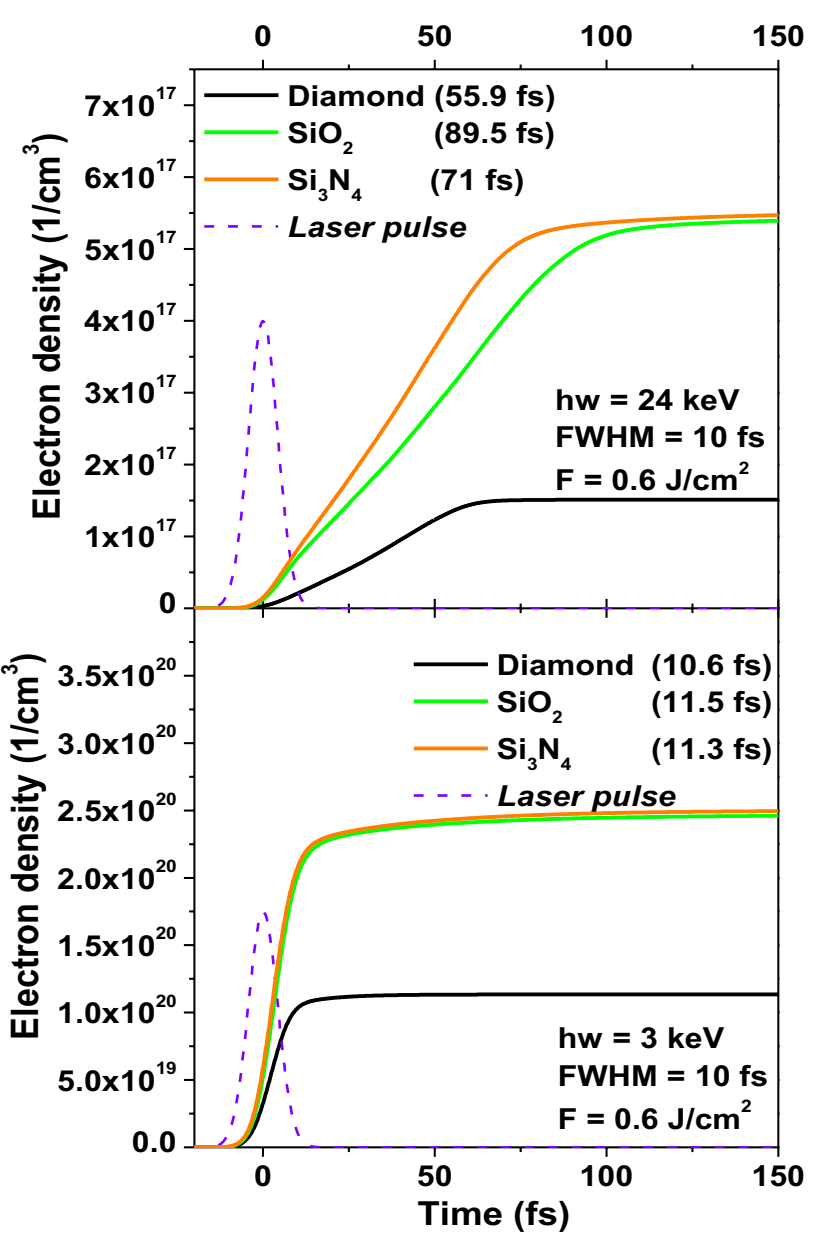

Update to Fig. 3 from the original article. Modeled electron-hole pair density in diamond, $\mathrm{SiO}_{2}$ and $\mathrm{Si}_{3} \mathrm{~N}_{4}$ irradiated with a 10 fs laser pulse of $0.6 \mathrm{~J} / \mathrm{cm}^{2}$ fluence. The case of $24 \mathrm{keV}$ photon energy is shown on the top-panel, and of $3 \mathrm{keV}$ photon energy is on the bottom-panel. FWHM of electron density increase is shown for each materials in the legend of the figures. A laser pulse profile is schematically added as a dashed line

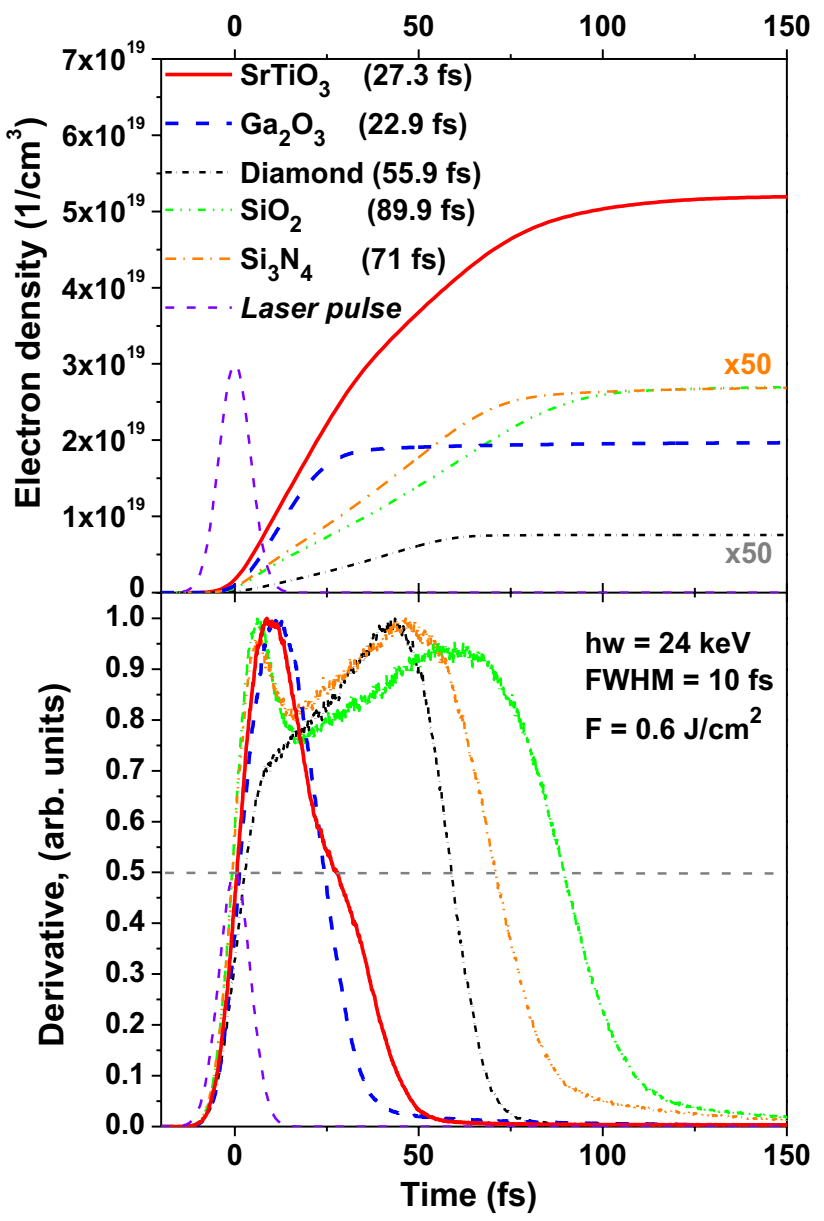

Update to Fig. 6 from the original article. Calculated electron-hole pair density in $\mathrm{SrTiO}_{3}$ and $\mathrm{Ga}_{2} \mathrm{O}_{3}$ irradiated with a 10 fs laser pulse of $0.6 \mathrm{~J} / \mathrm{cm}^{2}$ fluence and $24 \mathrm{keV}$ photon energy (top-panel). The results for diamond, $\mathrm{SiO}_{2}$ and $\mathrm{Si}_{3} \mathrm{~N}_{4}$ are copied from the Fig. 3 for comparison; note, that the data for these materials are multiplied by 50 in order to be displayed on the same scale with the new materials. FWHM of electron density increase is shown for each material in the legend of the figure. The corresponding time derivatives of the electron densities increase for these materials (bottom-panel). A laser pulse envelope is schematically shown as a dashed line 


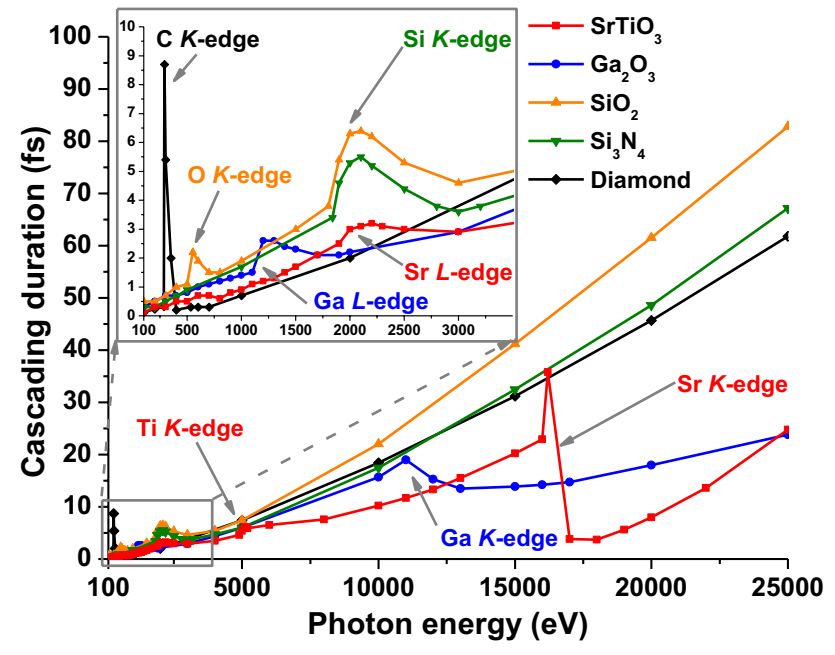

Update to Fig. 7 from the original article. Calculated FWHM of the electron-hole pair density increase in diamond, $\mathrm{SiO}_{2}, \mathrm{Si}_{3} \mathrm{~N}_{4}, \mathrm{SrTiO}_{3}$ and $\mathrm{Ga}_{2} \mathrm{O}_{3}$ irradiated with a 1 fs laser pulse for different photon energies. The inset zooms into the low photon energy range

\section{Reference}

1. K. Mecseki et al., Appl. Phys. Lett. 113, 114102 (2018)

Publisher's Note Springer Nature remains neutral with regard to jurisdictional claims in published maps and institutional affiliations. 